\title{
Primary Hypertrophic Osteoarthropathy Mimicking Juvenile Idiopathic Arthritis: A Novel SLCO2A1 Mutation and Imaging Findings
}

\author{
Murat Torgutalp ${ }^{\mathrm{a}}$ Ceren D. Durmaz ${ }^{\mathrm{b}}$ Halil G. Karabulut ${ }^{\mathrm{b}}$ Wenke Seifert $^{\mathrm{c}}$ \\ Denise Horn $^{d}$ Zehra Akkaya ${ }^{\mathrm{e}}$ Murat Turgay ${ }^{\mathrm{a}}$ \\ a Division of Rheumatology, Department of Internal Medicine, Faculty of Medicine, Ankara University, Ankara,

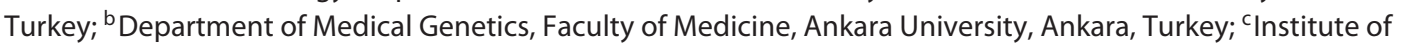 \\ Vegetative Anatomy and Institute of Cell Biology and Neurobiology, Charité - Universitätsmedizin Berlin, Berlin, \\ Germany; ${ }^{d}$ Institute for Medical and Human Genetics, Charité - Universitätsmedizin Berlin, Berlin, Germany; \\ eDepartment of Radiology, Faculty of Medicine, Ankara University, Ankara, Turkey
}

\section{Established Facts}

- Primary hypertrophic osteoarthropathy (PHO; pachydermoperiostosis) is a rare autosomal recessive disorder with systemic involvement caused by abnormalities in prostaglandin metabolism.

- $\mathrm{PHO}$ has 2 known genetic causes, namely HPGD or SLCO2A1 gene mutations.

- HPGD encodes the 15-hydroxyprostaglandin dehydrogenase that is the main enzyme of prostaglandin degradation. SLCO2A1 encodes a prostaglandin transporter that mediates the uptake and clearance of prostaglandins. High levels of prostaglandin E2 may assume a key role in disease pathogenesis.

\section{Novel Insights}

- PHO should be kept in mind in the differential diagnosis of inflammatory arthritis that is resistant to multiple immunosuppressive therapies.

- A novel SLCO2A1 mutation, p.Ile192Met, located within the 4th transmembrane domain was detected. This residue is conserved in the SLCO2A1 homologs of different species.

\section{Keywords}

Arthritis · Imaging · Pachydermoperiostosis · Primary

hypertrophic osteoarthropathy $\cdot$ SLCO2A1

\section{Abstract \\ Primary hypertrophic osteoarthropathy (PHO), also known as pachydermoperiostosis, is a rare, multisystemic, autoso-}

mal recessive condition typically presenting with digital clubbing, osteoarthropathy, and various skin manifestations. Radiographs show distinctive periosteal reaction and thickening along the long bones. PHO is caused by homozygous mutations in the HPGD gene in chromosome $4 q 34.1$ or the SLCO2A1 gene in 3q22.1q22.2. Here, we report on a 20-year-old male with enlarged and swollen joints with arthralgia, palmoplantar hyperhidrosis, and large hands and

\section{KARGER}

(c) 2019 S. Karger AG, Basel

E-Mail karger@karger.com

www.karger.com/cgr
Murat Torgutalp

Division of Rheumatology, Department of Internal Medicine

Faculty of Medicine, Ankara University

Ankara 06100 (Turkey)

E-Mail mtorgutalp@gmail.com 
feet with marked digital clubbing. We also present radiographic, MRI, and ultrasonographic features of the case. These clinical and imaging findings were compatible with the diagnosis of $\mathrm{PHO}$, and a novel homozygous mutation, c.576C>G, p.lle192Met, was found in SLCO2A1.

(c) 2019 S. Karger AG, Basel

Primary hypertrophic osteoarthropathy (PHO; MIM 259100 and 614441) is an uncommon autosomal recessive condition with multisystem involvement caused by mutations in the HPGD or SLCO2A1 genes [Uppal et al., 2008; Zhang et al., 2012]. The characteristic features of this disorder are skin manifestations like pachydermia, seborrhoeic dermatitis, and thickened/furrowed skin, as well as digital clubbing, osteoarthropathy, periostitis of tubular bones, and synovial effusions which can interfere with other causes of arthritis [Castori et al., 2005]. Plain radiographs exhibit periosteal thickening along the diaphysis of the long bones that are affected centripetally during the course of the disease progress. Furthermore, bone resorption like acroosteolysis can be seen [Pineda et al., 1987; Martinez-Lavin, 2011]. Magnetic resonance imaging (MRI) findings of some $\mathrm{PHO}$ cases portraying diaphyseal cortical bone and endosteal abnormalities, periostitis, and convoluted intraosseous vascular channels have been reported [Adams et al., 2016].

HPGD, located in $4 \mathrm{q} 34.1$, is one of the genes known to cause this phenotype. HPGD consists of 7 exons and encodes the 15-hydroxyprostaglandin dehydrogenase that is the main enzyme of prostaglandin degradation. On the other hand, SLCO2A1 is located at 3q22.1q22.2 and encodes a prostaglandin transporter (PGT) which plays a significant role in mediating the uptake and clearance of prostaglandins. High levels of prostaglandins, especially prostaglandin E2, may assume a key part in disease pathogenesis. While homozygous or compound heterozygous mutations in the HPGD gene cause autosomal recessive PHO-1, SLCO2A1 mutations are responsible for the autosomal recessive $\mathrm{PHO}-2$. The genotype-phenotype correlation is difficult to establish for PHO. However, mutations in SLCO2A1 have been distinguished as the primary causative factor of PHO in East Asians [Sasaki et al., 2012].

Here, we present a $\mathrm{PHO}$ patient with a novel SLCO2A 1 mutation who initially had been diagnosed with juvenile idiopathic arthritis (JIA).
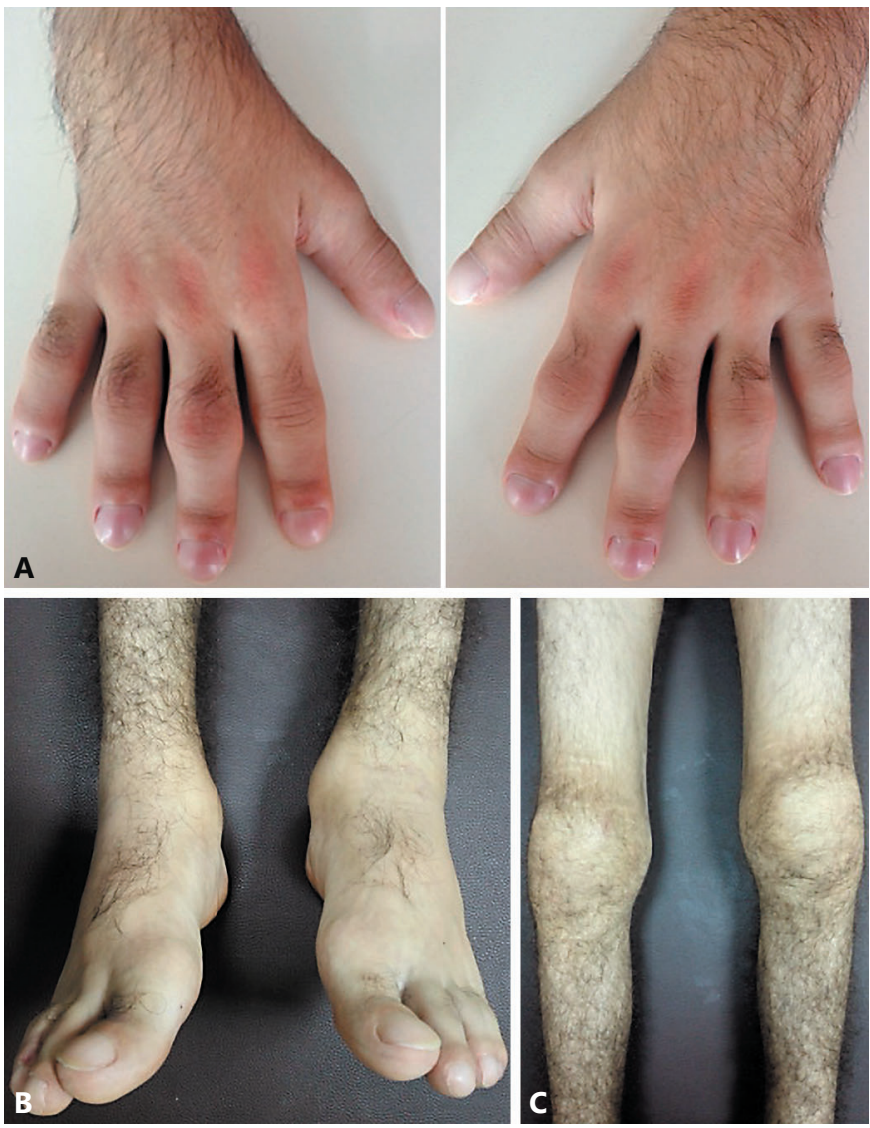

Fig. 1. Digital clubbing of fingers (A) and toes (B). Swollen hands (A), left ankle (B), and bilateral knee joints (C).

\section{Case Report}

A 20-year-old male was admitted to our Rheumatology department at Ibn-i Sina Hospital of Ankara University in March 2017. His main complaints were diffuse joint pain, swelling, and an hour of morning stiffness. These problems started at the age of 12, and the patient consulted several local hospitals and received indistinct treatments mostly nonsteroidal anti-inflammatory drugs which did not relieve the symptoms completely. Thereafter, he was diagnosed somewhere else with JIA. In the following years, continuous relapses with joint inflammation required intraarticular steroid injections, immunosuppressive treatments (prednisolone and methotrexate), and tumor necrosis factor- $\alpha$ blockers (adalimumab and etanercept) with unremarkable efficacy.

On clinical examination, we discovered thick skin with seborrheic eczema and hyperhidrosis of palms and soles. He had hand, feet, and knee swelling, and his hands and feet examination indicated clubbing of all digits (Fig. 1). Cardiac, pulmonary, and abdominal examinations were within normal range. Superficial lymph nodes, liver, and spleen were not palpable. He had swelling of proximal interphalangeal and metacarpophalangeal joints, wrists, knees, and ankles. Laboratory investigation revealed normal complete blood count as well as normal serum creatinine and 

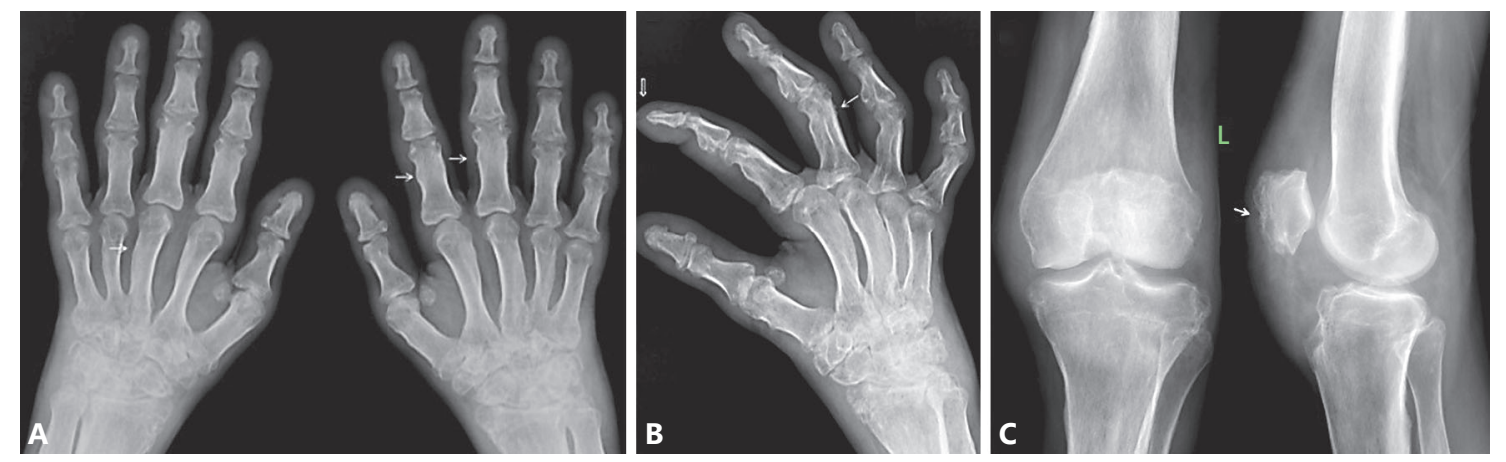

Fig. 2. A Plain radiographs show marked erosive changes in the wrist joints as well as thickening in the mid-diaphyseal parts of metacarpal and phalangeal bones, mostly marked on the proximal phalanges (arrows). B Oblique radiograph of the right hand shows the bowing deformity and thickening of the tubular bones of the hand and subperiosteal new bone formation as irregularity on the

dorsal aspect of the 3rd middle phalanx (arrow), as well as extensive erosive changes at the wrist joint. Clubbing at the finger tips is also notable (open arrow). C Lateral radiographs of the left knee joint demonstrate soft tissue swelling and cortical thickening on metaphysis of distal femur and proximal tibia, also particularly noticeable on the anterior cortex of the patella (arrow).
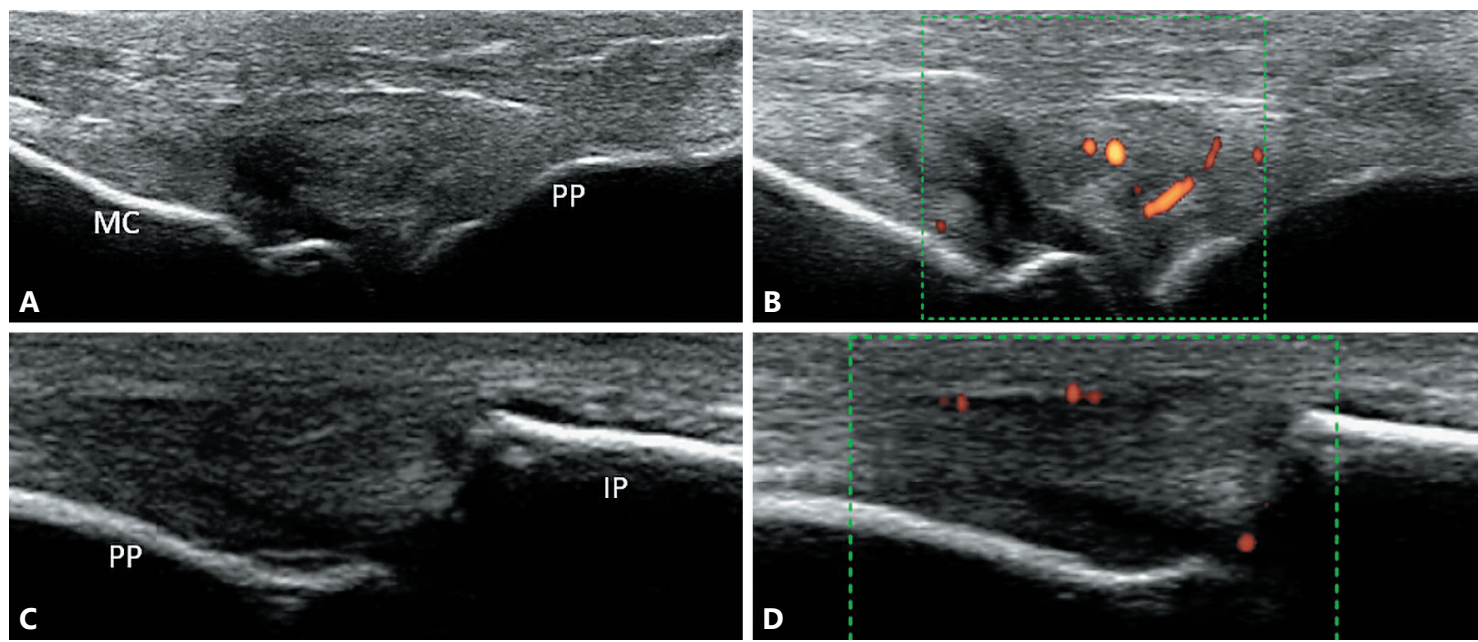

Fig. 3. Ultrasound findings of the right 3rd metacarpophalangeal $(\mathbf{A}, \mathbf{B})$ and right $3 \mathrm{rd}$ proximal interphalangeal (C, D) joints. Marked synovial hypertrophy and effusion $(\mathbf{A}, \mathbf{C})$ and power Doppler sig- nal activity (B, D). MC, metacarpal bone; PP, proximal phalanx; IP, intermediate phalanx. transaminase levels. Rheumatologic serologic tests were normal; rheumatoid factor, anti-cyclic citrullinated peptide, anti-nuclear antibody, anti-extractable nuclear antigens, anti-dsDNA, and anti-neutrophil cytoplasmic antibodies were all negative. C-reactive protein level was $57 \mathrm{mg} / \mathrm{L}$, erythrocyte sedimentation rate was $36 \mathrm{~mm} / \mathrm{h}$. Endocrinological evaluation was also normal; basal growth hormone levels, insulin-like growth factor 1, parathyroid hormone, serum $25-\mathrm{OH}$ vitamin $\mathrm{D}$, adrenocorticotropic hormone, cortisol, thyroid stimulating hormone, fT3, fT4, luteinizing hormone, follicle stimulating hormone, and testosterone levels were within normal ranges (Table 1). Prostaglandin E2 (PGE2) levels of serum and urine were not available. Echocar- diography and arterial blood gas analysis were normal. Tumor biomarkers were negative. A broad malignancy workup, which included serum and urine protein electrophoresis as well as neckchest-abdomen-pelvis computed tomography, was performed, and all were negative.

His parents asserted not to be related although they came from the same rural town. He noted that his father and uncle (mother's brother) both had similar looking digits without any other clinical findings. His father was also found to have digital clubbing of both upper and lower extremities.

Joint radiographs were similarly in accordance with inflammatory arthritis (especially erosions in wrists), but also revealed thick- 

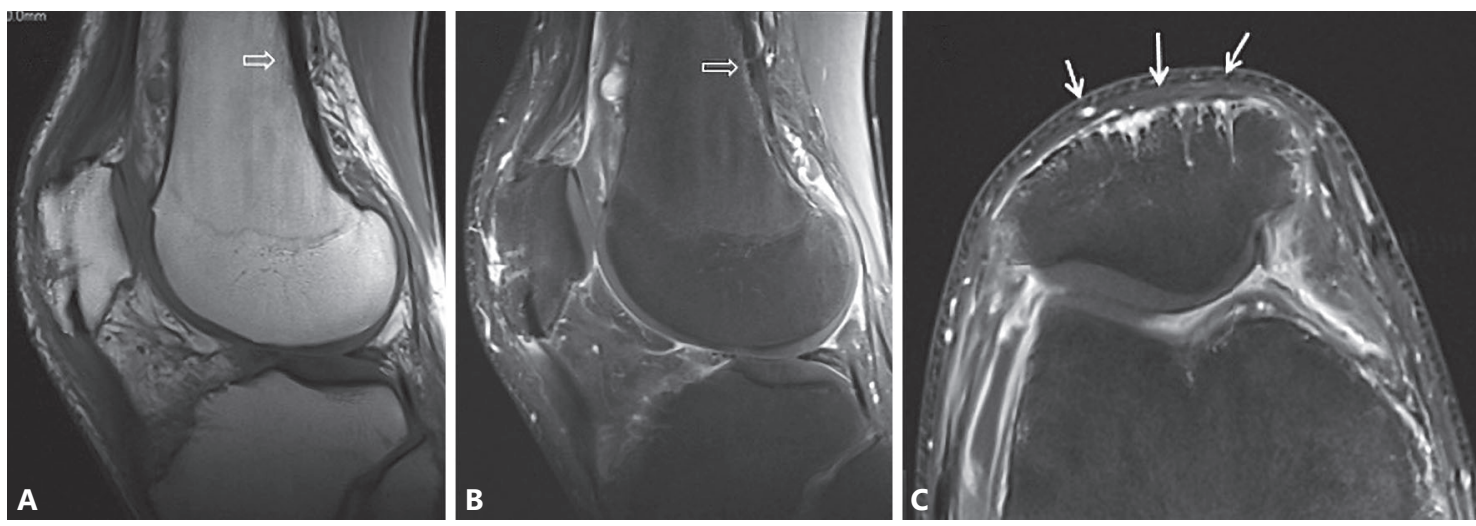

Fig. 4. A, B Hypertrophic subperiosteal new bone formation and cortical irregularity with increased periosteal vascularity are depicted in the sagittal T1-weighted (A), and the corresponding fatsuppressed post-contrast T1-weighted (B) images. The posterior cortex of the distal femur is thick ( $\mathbf{A}$, open arrow), and endosteal bone shows increased vascularity at this location (B, open arrow). C Periosteal linear enhancement and increased subcortical vascularity (arrows) are better visible on the fat-suppressed T1-weighted axial image.

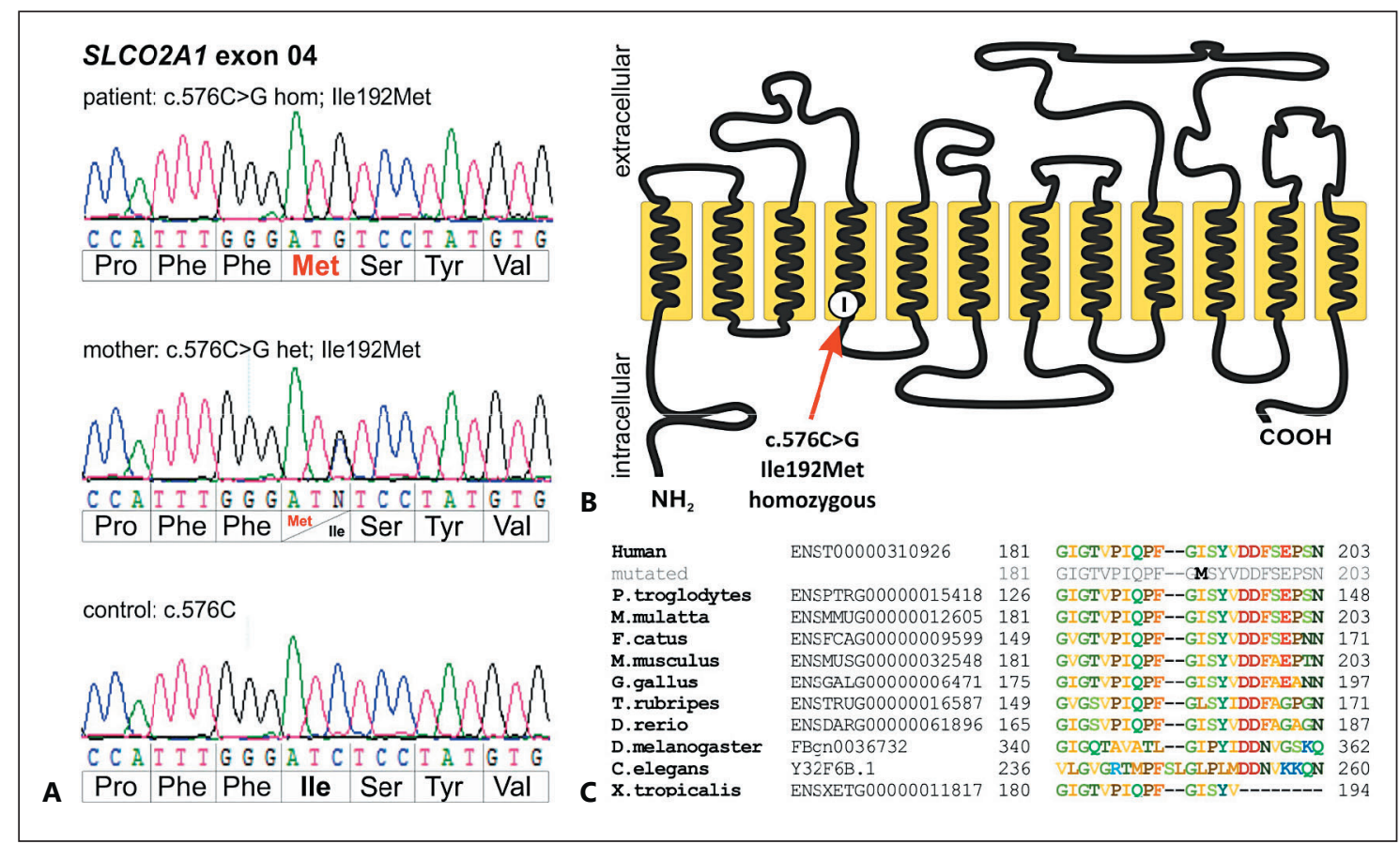

Fig. 5. A Electropherograms of the proband, his mother, and a healthy control, showing a novel homozygous c.576C $>\mathrm{G}$ mutation, p.Ile192Met, in exon 4 of the SLCO2A1 gene of the proband. His mother is heterozygous for the same mutation. B Schematic presentation of the prostaglandin transporter protein and the missense mutation of our patient. C Conservation of these residues in the SLCO2A1 homologs from different species. ening in the mid-diaphyseal parts of the metacarpal and phalangeal bones, and cortical thickening on metaphysis of distal femur and proximal tibia (Fig. 2). Ultrasound of the 3rd metacarpophalangeal and the 3rd proximal interphalangeal joints showed synovial hypertrophy and synovial effusion with increased vascularity on power Doppler, all consistent with inflammatory arthritis (Fig. 3). Hypertrophic subperiosteal new bone formation and cortical irregularity with increased periosteal vascularity were determined by MRI of the femur (Fig. 4). 
Based on the clinical and radiological findings, and after the exclusion of the other possible etiologies, the patient was diagnosed with PHO. The patient's medication with adalimumab and methotrexate was substituted for acemetacin. Excellent therapeutic response was achieved within only 2 weeks. Besides, during follow-up for 18 months, the patient's complaints disappeared, erythrocyte sedimentation rate and C-reactive protein values dropped to normal levels, and Doppler activity in ultrasound disappeared.

\section{Methods}

Genomic DNA was isolated from peripheral blood samples of the patient and his unaffected mother using MagnaPure LC DNA Isolation Kit-Large Volume and MagnaPure LC instrument (Roche Applied Science, Mannheim, Germany). All exons and the flanking regions of the HPGD and the SLCO2A 1 genes were amplified by PCR, and sequence analysis was performed using BigDye Terminator v3.1 Cycle Sequencing Kit on an ABI PRISM $3130 \mathrm{Ge}$ netic Analyzer (Thermo Fisher Scientific, Waltham, MA, USA). Primers used in PCR and sequencing reactions are shown in online supplementary Tables 1 and 2 (for all online suppl. material, see www.karger.com/doi/10.1159/000500988).

\section{Results}

We detected a novel homozygous c.576C $>$ G mutation, p.Ile192Met, in exon 4 of the SLCO2A1 gene in the proband. His mother was found to be heterozygous for the same mutation (Fig. 5A). We could not perform molecular testing of his father due to social issues. This variant was listed neither in the 1000 Genomes nor in the ExAC database (http://browser.1000genomes.org/index.html and http://exac.broadinstitute.org/, respectively). The impact of the mutation on PGT structure and function predicted by in silico analysis was described as damaging.

\section{Discussion}

$\mathrm{PHO}$ is characterized by pachydermia, clubbing of the digits, and periostosis [Castori et al., 2005]. The disease usually starts in childhood or adolescence, and there is an insidious onset of joint pain, swelling of the joints, and enlargement of the extremities with skin findings. Although rheumatologic findings like arthralgia and arthritis are present in $20-40 \%$ of patients, massive articular effusion with joint dysfunction is very rare [Cooper et al., 1992].

Our patient was initially seen in a local hospital because of pain and swelling of the joints and received vari-
Table 1. Clinical laboratory test results

\begin{tabular}{ll}
\hline Test & Result (reference range) \\
\hline Complete blood count & \\
Hemoglobin, g/dL & $13.5(13.2-17.3)$ \\
White blood cell count, $\times 10^{9} / \mathrm{L}$ & $7.57(4.5-11)$ \\
Platelet count, $\times 10^{9} / \mathrm{L}$ & $361(150-400)$ \\
\hline Serum biochemistry tests & \\
Creatinine, mg/dL & $0.47(0.7-1.3)$ \\
Alanine aminotransferase, U/L & $5(10-49)$ \\
Aspartate aminotransferase, U/L & $11(<34)$ \\
\hline Rheumatologic and immunological markers & \\
Rheumatoid factor, IU/mL & $<9.3(0-14)$ \\
Anti-cyclic citrullinated peptide, U/mL & $17.2(0-20)$ \\
Anti-nuclear antibody titer $(\mathrm{ANA})$ & $<1: 80$ \\
Anti-extractable nuclear antigens $(\mathrm{ENA})$ & Negative \\
Anti-dsDNA, IU/mL & $3.8(0-40)$ \\
Anti-neutrophil cytoplasmic antibody & Negative \\
\hline Serum capillary protein electrophoresis & \\
Albumin, \% & $47(55.8-66.1)$ \\
Alpha-1-globulin, \% & $7.3(2.9-4.9)$ \\
Alpha-2-globulin, \% & $11.5(7.1-11.8)$ \\
Beta globulin, \% & $10.6(8.4-13.1)$ \\
Gamma globulin, \% & $23.6(11.1-18.8)$ \\
\hline Urine protein electrophoresis & Normal \\
\hline Endocrinological evaluation & \\
Growth hormone, ng/mL & \\
Insulin-like growth factor-1, ng/mL & $160(219-644)$ \\
Adrenocorticotropic hormone, morning, & \\
pg/mL & $17.45(7.2-63.3)$ \\
Thyroid stimulating hormone, $\mu \mathrm{IU} / \mathrm{mL}$ & $2.16(0.55-4.78)$ \\
Free T3, pmol/L & $5.62(3.5-6.5)$ \\
Free T4, pmol/L & $16.01(11.5-22.7)$ \\
Luteinizing hormone, mIU/mL & $7.62(1.5-9.3)$ \\
Follicle stimulating hormone, mIU/mL & $1.96(1.4-18.1)$ \\
Prolactin, ng/mL & $16.15(2.1-17.7)$ \\
Testosterone, male, ng/dL & $285(175-781)$ \\
Parathyroid hormone, pg/mL & $48(12-88)$ \\
25-OH vitamin D3, winter, $\mu \mathrm{g} / \mathrm{L}$ & $15(10-60)$ \\
\hline
\end{tabular}

ous immunosuppressive medications, including biological treatments, with the diagnosis of JIA. However, in our clinical examination, JIA was excluded by laboratory tests and imaging findings, whereas the presence of clubbing and skin signs in a young individual with no underlying lung and liver disease, or malignancy, was powerfully indicative of PHO. This diagnosis was supported by the radiological findings.

In a study of 76 patients by Jajic et al. [2001], periosteal reaction was found throughout long bones in all patients, and acroosteolysis was detected in $23.7 \%$ of them [Jajic et al., 2001; Martinez-Lavin, 2011]. In the presented case, the X-ray characteristics like subperiosteal new bone formation, thickening of cortex on metaphysis, cortical 
irregularity with increased periosteal vascularity were distinctive for PHO. The identified MRI features and histological findings are compatible with each other [Loredo et al., 1996; Pineda, 1992]. In accordance with the reported MRI findings in the literature, our case exhibited periosteal thickening and endosteal enhancement on postcontrast images compatible with increased subcortical vascularity and tortuous intraosseous vascular channels [Pineda, 1992; Loredo et al., 1996; Adams et al., 2016].

$S L C O 2 A 1$ is a member of the organic anion transporting polypeptide superfamily (OATPs). OATPs consist of 12 transmembrane domains and mediate the transport of a broad range of endo- as well as xenobiotics [Hagenbuch and Stieger, 2013]. SLCO2A1 was mapped to 3q22.1q22.2, is composed of 14 exons, and encodes a 643-amino acids protein, also known as PGT [Lu et al., 1996]. PGT involves PGE2 clearance. PGE2 metabolic clearance occurs via 2 steps: (1) PGT-mediated uptake across the plasma membrane, and (2) 15-hydroxyprostaglandin dehydrogenase-mediated degradation [Nomura et al., 2004]. In the case of homozygous mutations in any of the genes encoding these 2 proteins, PGE2 levels increase and lead to the features of PHO. Like all other OATP proteins, PGT is an evolutionarily well-conserved protein and has 12 transmembrane domains. The p.Ile192Met mutation is located within the 4 th transmembrane domain (Fig. 5B). This residue is conserved within the SLCO2A1 homologs of different species (Fig. 5C). SIFT and PolyPhen-2 analysis showed that this mutation is damaging with a SIFT score of 0.00 and probably damaging with a PolyPhen-2 score of 1.000. Nonsense, missense, frameshift, splice site, and indel mutations in the $S L C O 2 \mathrm{~A} 1$ gene were reported in PHO. However, as the novel variant identified in our case, most of the PHO-causing mutations in SLCO2A1 are missense type [Guo et al., 2017]. We found our patient to be homozygous for p.Ile192Met, and his mother was found to be heterozygous for the same mutation. We could not evaluate his father's DNA. Although his parents claimed not to be related, isolated digital clubbing in his father suggested that he might be heterozygous for the same mutation. In the literature, some patients with isolated digital clubbing have been reported to have a heterozygous mutation in SLCO2A 1 [Diggle et al., 2012].

\section{Conclusion}

In conclusion, we identified a novel missense mutation in the SLCO2A1 gene in a PHO patient and present this case with different imaging modalities like radiography, MRI, and ultrasound. In addition, PHO should be kept in mind in the differential diagnosis of inflammatory arthritis resistant to multiple immunosuppressive therapies, although it is a rare disease.

\section{Acknowledgements}

The authors would like to thank the patient and his family for their collaboration.

\section{Statement of Ethics}

Informed consent was obtained from the patient and his mother. The authors have no ethical conflicts to disclose.

\section{Disclosure Statement}

The authors have no conflicts of interest to declare.

\section{References}

\footnotetext{
Adams B, Amin T, Leone V, Wood M, Kraft JK: Primary hypertrophic osteoarthropathy: ultrasound and MRI findings. Pediatr Radiol 46:727-730 (2016).

Castori M, Sinibaldi L, Mingarelli R, Lachman RS, Rimoin DL, Dallapiccola B: Pachydermoperiostosis: an update. Clin Genet 68:477-486 (2005).

-Cooper RG, Freemont AJ, Riley M, Holt PJ, Anderson DC, Jayson MI: Bone abnormalities and severe arthritis in pachydermoperiostosis. Ann Rheum Dis 51:416-419 (1992).

Diggle CP, Parry DA, Logan CV, Laissue P, Rivera $\mathrm{C}$, et al: Prostaglandin transporter mutations cause pachydermoperiostosis with myelofibrosis. Hum Mutat 33:1175-1181 (2012)

Guo T, Yang K, Liu L, Tan ZP, Luo H: Identification of two novel mutations in the SLCO2A1 prostaglandin transporter gene in a Chinese patient with primary hypertrophic osteoarthropathy. Mol Med Rep 15:2977-2982 (2017).

\section{Hagenbuch B, Stieger B: The SLCO (former} SLC21) superfamily of transporters. Mol As-

Jajic Z, Jajic I, Nemcic T: Primary hypertrophic osteoarthropathy: clinical, radiologic, and scintigraphic characteristics. Arch Med Res 32:136-142 (2001).

Loredo R, Pathria MN, Salonen D, Resnick D: Magnetic resonance imaging in pachydermoperiostosis. Clin Imaging 20:212-218 (1996).

Lu R, Kanai N, Bao Y, Schuster VL: Cloning, in vitro expression, and tissue distribution of a human prostaglandin transporter cDNA(hPGT). J Clin Invest 98:1142-1149 (1996).
} pects Med 34:396-412 (2013). 
Martinez-Lavin M: Miscellaneous non-inflammatory musculoskeletal conditions. Pachydermoperiostosis. Best Pract Res Clin Rheumatol 25:727-734 (2011).

- Nomura T, Lu R, Pucci ML, Schuster VL: The two-step model of prostaglandin signal termination: in vitro reconstitution with the prostaglandin transporter and prostaglandin 15 dehydrogenase. Mol Pharmacol 65:973-978 (2004).
Pineda C: Diagnostic imaging in hypertrophic osteoarthropathy. Clin Exp Rheumatol 10 Suppl 7:27-33 (1992).

Pineda CJ, Martinez-Lavin M, Goobar JE, Sartoris DJ, Clopton P, Resnick D: Periostitis in hypertrophic osteoarthropathy: relationship to disease duration. AJR Am J Roentgenol 148: 773-778 (1987).

Sasaki T, Niizeki H, Shimizu A, Shiohama A, Hirakiyama $A$, et al: Identification of mutations in the prostaglandin transporter gene $S L$ $\mathrm{CO} 2 \mathrm{~A} 1$ and its phenotype-genotype correlation in Japanese patients with pachydermoperiostosis. J Dermatol Sci 68:36-44 (2012).
Uppal S, Diggle CP, Carr IM, Fishwick CW, Ahmed M, et al: Mutations in 15-hydroxyprostaglandin dehydrogenase cause primary hypertrophic osteoarthropathy. Nat Genet 40:789-793 (2008).

Zhang Z, Xia W, He J, Zhang Z, Ke Y, et al: Exome sequencing identifies SLCO2A1 mutations as a cause of primary hypertrophic osteoarthropathy. Am J Hum Genet 90:125-132 (2012). 